\title{
Overexpression of microRNA-125a-3p effectively inhibits the cell growth and invasion of lung cancer cells by regulating the mouse double minute 2 homolog/p53 signaling pathway
}

\author{
SHENGLEI LI ${ }^{1 *}$, XIN LI $^{2 *}$, HUASI ZHAO $^{3}$, MING GAO $^{2}$, FENG WANG $^{2}$ and WENCAI LI \\ Departments of ${ }^{1}$ Pathology, ${ }^{2}$ Oncology and ${ }^{3}$ Respiratory, \\ The First Affiliated Hospital of Zhengzhou University, Zhengzhou, Henan 450052, P.R. China
}

Received May 12, 2014; Accepted January 29, 2015

DOI: $10.3892 / \mathrm{mmr} .2015 .4038$

\begin{abstract}
MicroRNAs (miRs) are a family of small noncoding RNAs that are 21-24 nucleotides in length. Decreased expression of hsa-miR-125a-3p is observed in a number of patients with non-small cell lung cancer; however, it is not clear how this miRNA regulates the growth and invasion of lung tumor cells. The aim of the present study was to identify the function of hsa-miR-125a-3p in the growth and invasion of lung cancer cells. The expression of hsa-miR-125a-3p in the A549, NCI-H460 and SPCA-1 lung cancer cell lines was analyzed by reverse transcription-quantitative polymerase chain reaction and the human bronchiolar epithelium cell line (HBE) was used as a control. The results demonstrated that the expression of hsa-miR-125a-3p was significantly lower in NCI-H460, A549 and SPCA-1 cells, compared with that in HBE cells. Overexpression of sense miR-125a-3p in the A549 lung cancer cell line inhibited cell proliferation for 5-7 days $(\mathrm{P}<0.01)$, and transfection of antisense miR-125a-3p did not suppress the cell growth of the lung cancer cells. In addition, overexpression of miR-125a-3p in the NCI-H460 lung cancer cell line markedly induced cell apoptosis, which was detected by fluorescence-activated cell sorting with annexin V-fluorescein isothiocyanate/propidium iodide staining. The results of the Transwell migration assay also revealed that transfection of $\mathrm{miR}-125 \mathrm{a}-3$ p resulted in decreased
\end{abstract}

Correspondence to: Mr. Feng Wang, Department of Oncology, The First Affiliated Hospital of Zhengzhou University, 1 JianSheDongLu, Zhengzhou, Henan 450052, P.R. China

E-mail: wangfeng_as@163.com

Mr. Wencai Li, Department of Pathology, The First Affiliated Hospital of Zhengzhou University, 1 JianSheDongLu, Zhengzhou, Henan 450052, P.R. China

E-mail: wencaiali9706@163.com

${ }^{*}$ Contributed equally

Key words: hsa-miR-125a-3p, lung cancer, p53, mouse double minute-2 migration of lung cancer tumor cells. The pro-apoptotic gene p53 expression was detected by western blot analysis. The results revealed that the expression of mouse double minute (MDM)-2 homolog, the principal cellular antagonist of p53, was decreased and p53 expression was upregulated in sense has-miR-125a-3p transfected A549 cells. This was consistent with that observed in NCI-H460 cells, suggesting that hsa-miR-125a-3p may be involved in the regulation of the MDM2/p53 signaling pathway in lung cancer cells. In conclusion, overexpression of hsa-miR-125a-3p significantly inhibited the proliferation and invasion of lung cancer cells, which may aid in determining the mechanisms underlying the development of lung cancer.

\section{Introduction}

MicroRNAs (miRNAs) are endogenously encoded small noncoding RNAs, which are 21-24 nucleotides long and regulate posttranscriptional mRNA expression (1). miRNA has been widely observed in various organisms. One-third of human genes can be regulated by the miRNAs (2). miRNAs are important in translational inhibition or destabilization of the target mRNA. It is reported that $>50 \%$ of miRNA genes are located in cancer-related genomic regions or fragile sites, suggesting that miRNAs may be important in the progression and development of different types of tumors $(3,4)$.

miR-125a is a member of the miRNA family, it has a precursor form and two mature forms (miR-125a-3p and miR-125a-5p) (5). The mature forms have biological activity, and the mature microRNA hsa-miR-125a-3p is derived from the $3^{\prime}$ end of pre-miR-125a. Jiang et al (6) demonstrated that hsa-miR-125a-3p and hsa-miR-125a-5p were downregulated in non-small cell lung cancer and have inverse effects on the invasion and migration of lung cancer cells. Huang et al (7) reported that miRNA-125a-3p is a negative regulator of the Ras homolog gene family (Rho)A-actomyosin pathway in A549 cells, and loss of miR-125a-3p leads to increased migration of tumor cells due to the upregulation of RhoA expression.

Lung cancer is a common disease with highest incidence and mortality (8-10). Small-cell lung carcinoma is one of the main types of lung cancer $(11,12)$. The 5-year survival rate 
for lung cancer is only $10 \%(13,14)$. The tumour suppressor p53 is known to prevent cancer progression by inhibiting proliferation and inducing apoptosis of tumor cells $(15,16)$. Mouse double minute 2 homolog (MDM2), also termed E3 ubiquitin-protein ligase $(17,18)$, is an important negative regulator of the p53 tumor suppressor. Mdm2 protein recognizes the N-terminal trans-activation domain of the p53 tumor suppressor and inhibits p53 transcriptional activity $(19,20)$. The p53-MDM2 signaling pathway is known to control cancer invasion and metastasis (21). In the present study, the expression of miR-125a-3p was detected in three lung cancer cell lines, and the effects of miR-125a-3p on the proliferation and migration of lung cancer cells were determined.

\section{Materials and methods}

Cells lines. A549, NCI-H460 and SPCA-1 human lung cancer cell lines obtained from the American Type Culture Collection (Manassas, VA, USA) were cultured in Dulbecco's modified Eagle's medium (DMEM) supplemented with $10 \%$ fetal bovine serum (FBS) (GE Healthcare Life Sciences, Logan, UT, USA).

MTT assay. The A549 lung cancer cell line was plated onto 48-well plates. Following adherence of the cells for $8 \mathrm{~h}$, sense hsa-miR-125a-3p and anti-sense hsa-miR-125a-3p was transfected into A549 cells. The cells were cultured for 1, 3, 5 and 7 days, respectively. Then, cell proliferation was evaluated by an MTT assay (Sigma-Aldrich, St. Louis, MO, USA). Briefly, $10 \mu \mathrm{l}$ of $5 \mathrm{mg} / \mathrm{ml} \mathrm{MTT}$ was added into the medium. After $4 \mathrm{~h}, 150 \mu \mathrm{l}$ dimethylsulfoxide (Sigma-Aldrich) was added for $15 \mathrm{~min}$. The plates were then read on a microplate reader (Thermo Fisher Scientific, Inc., Waltham, MA, USA) at a test wavelength of $490 \mathrm{~nm}$ and a reference wavelength of $570 \mathrm{~nm}$.

Reverse transcription-quantitative polymerase chain reaction (RT-qPCR). Total RNA was extracted from A549, NCI-H460 and SPCA-1 lung cancer cells and HBE normal cells by TRIzol reagent (Takara Bio Inc., Dalian, China), and cDNA was transcribed using the PrimeScript ${ }^{\circledR}$ RT reagent kit (Takara Bio Inc.) according to the manufacturer's instructions. RT-qPCR was performed to evaluate hsa-miR-125a-3p expression on ABI 7500 system. The following primer sequences were used: Sense, 5'-ACAGGUGAGGUUCUUGGG AGCC-3' and antisense, 5'-GGCUCCCAAGAACCUCAC CUGU-3' for hsa-miR-125a-3p; and sense, 5'-CTCGCTTCG GCAGCACA-3' and antisense, 5'-AACGCTTCACGAATT TGCGT-3' for small nuclear RNA U6RNA, which was used as a reference gene. The primers were synthesized by Quanshijin Corporation (Beijing, China). PCR thermal cycling conditions were as follows: 40 cycles of $12 \mathrm{sec}$ at $95^{\circ} \mathrm{C}$ and $1 \mathrm{~min}$ at $60^{\circ} \mathrm{C}$ using SYBR Green (Takara Bio Inc.).

Western blot analysis. The A549 and NCI-H460 lung cancer cell lysates were prepared and separated by SDS-PAGE (22-24). After transferring the protein from the gel to the membrane, the monoclonal mouse anti-human p53 (DO-2; sc-53394), MDM-2 (SMP14; sc-965) and $\beta$-actin (C-2; sc-8432) primary antibodies were used for incubation. The cells were then incubated with a horseradish peroxidase-conjugated goat anti-mouse secondary antibody. All of the antibodies were obtained from Santa Cruz Biotechnology, Inc. (Santa Cruz, CA, USA). The western blots were visualized usin Immobilon Western Chemiluminescent HRP Substrate (ECL) (EMD Millipore, Billerica, MA, USA).

Flow cytometric analysis. The cell apoptosis rate was determined by flow cytometric analysis with annexin V-fluorescein isothiocyanate (FITC)/propidium iodide (PI) staining $(25,26)$. Briefly, $1 \times 10^{5}$ lung cancer cells were plated into 6 -well plates. After $6 \mathrm{~h}$, the cells were transfected with sense hsa-miR-125a-3p, anti-sense miR-125a-3p and scrambled miRNA, respectively, which were designed and synthesized by Shanghai GenePharma Co., Ltd. (Shanghai, China). After five days, cell apoptosis was detected by annexin V-FITC/PI (Abcam, Cambridge, MA, USA) dual staining analysis according to the protocols. Finally, the cells were detected and analyzed by flow cytometry on a FACSCalibur Cell Sorting system (BD Biosciences, Franklin Lakes, NJ, USA).

Transwell assay. A549 cells were cultured in DMEM with 10\% FBS and Transwell compartments (Corning Incorporated, New York, NY, USA) were prepared with 24-well format with an $8-\mu \mathrm{m}$ pore size. The assay was conducted using methods described previously $(27,28)$. Briefly, the lower compartment was filled with $2.6 \mathrm{ml} \mathrm{DMEM}$ with $0.5 \%$ FBS containing $40 \mu \mathrm{g} / \mathrm{ml}$ collagen I (Sigma-Aldrich). The Transwell insert was then added to the well by merging the bottom of the insert into the medium in the lower compartment. A549 cells $\left(1 \times 10^{5}\right)$ were then added to the upper compartment. The cells were incubated in the Transwell plate at $37^{\circ} \mathrm{C}$ and $5 \% \mathrm{CO}_{2}$ for $8 \mathrm{~h}$. Then, the insert was carefully removed. A549 cells on the lower side of the insert filter were quickly fixed in $5 \%$ glutaraldehyde for $10 \mathrm{~min}$, and stained with $1 \%$ crystal violet in $2 \%$ ethanol for $20 \mathrm{~min}$. Finally, excess crystal violet was removed by quickly merging the insert in $\mathrm{dd}_{2} \mathrm{O}$ for a few seconds and the number of cells on the lower side of the filter was counted under a microscope (CX22; Olympus, Tokyo, Japan).

Statistical analysis. Data were analyzed with Student's t-test using SPSS software, version 18.0 (SPSS, Inc., Chicago, IL, USA). The data are presented as the mean \pm standard error of the mean. $\mathrm{P}<0.01$ was considered to indicate a statistically significant difference.

\section{Results}

Relative mRNA levels of hsa-miR-125a-3p are detected in A549, NCI-H460 and SPCA-1 lung cancer cell lines. The expression of has-miR-125a-3p was detected by RT-qPCR in a panel of three colorectal cancer cell lines. The HBE cell line was used as a control. As shown in Fig. 1, the results demonstrated that the mRNA levels of has-miR-125a-3p in the cancer cell lines were significantly lower than those in the HBE cells, suggesting that has-125a-3p may be a tumor suppressor gene. Notably, the expression of has-miR-125a-3p in NCI-H460 cells was lowest, and most significantly different compared with the control cells $(\mathrm{P}<0.01)$. 


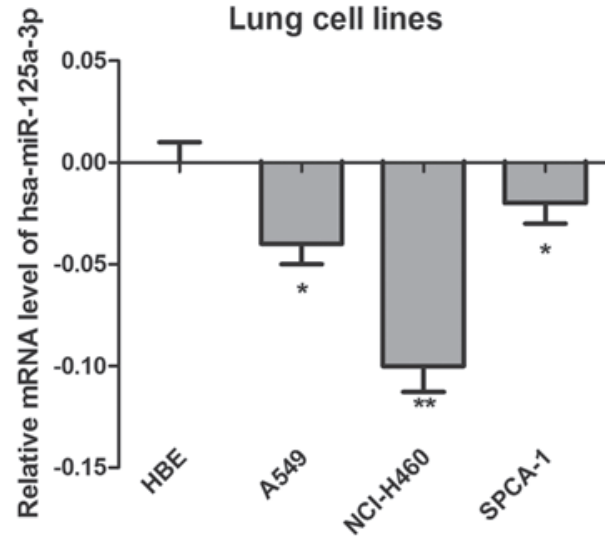

Figure 1. Histogram of relative mRNA levels of hsa-miR-125a-3p. The A549, NCI-H460 and SPCA-1 lung cancer cell lines were cultured and total RNA was extracted to detect the levels of hsa-miR-125a-3p by reverse transcription-quantitative polymerase chain reaction. The HBE human bronchiolar epithelium cell line was used as the normal control. ${ }^{*} \mathrm{P}<0.05$ and ${ }^{* *} \mathrm{P}<0.01$ vs. HBE.

Overexpression of sense hsa-miR-125a-3p inhibits the proliferation of A549 cells. Hsa-miR-125a-3p was overexpressed in A549 lung cancer cells, and an MTT assay was used to analyze the cell proliferation. As shown in Fig. 2, the lung cancer cells were transfected with sense hsa-miR-125a-3p or anti-sense has-miR-125a-3p for 1, 3, 5 or 7 days, respectively, and the results showed that transfection of sense hsa-miR-125a-3p inhibited the growth of A549 cells. In addition, overexpression of anti-sense hsa-miR-125a-3p did not suppress the proliferation of lung cancer cells.

Apoptosis rate increases in NCI-H460 lung cancer cells transfected with sense has-miR-125a-3p. In order to detect the effects of has-miR-125a-3p on cell apoptosis in lung cancer cells, cell apoptosis rates were detected by annexin V-FITC and PI dual staining analysis. The NCI-H460 lung cancer cell line was transfected with sense has-miR-125a-3p and anti-sense has-miR-125a-3p, and cultured for 5 days. The lung cancer cells transfected with scrambled miRNA were used as negative controls. As shown in Fig. 3, the apoptosis rate of sense has-miR-125a-3p transfected NCI-H460 cells was significantly higher than that of the controls $(\mathrm{P}<0.01)$.

Transfection of sense hsa-miR-125a-3p inhibits the cell migration and invasion of A549 lung cancer cells. In order to detect the effect of hsa-miR-125a-3p on cell migration and invasion of lung cancer cells, a Transwell migration assay was performed. As shown in Fig. 4, the number of migrated A549 cells was $33.50 \pm 2.27$ in the control group without any treatment. The number of migrated cells was $25.33 \pm 3.15$, which was less than that in the control group $(\mathrm{P}<0.01)$. Additionally, transfection of anti-sense hsa-miR-125a-3p significantly promoted cell migration compared with transfection with sense has-miR-125a-3p $(\mathrm{P}<0.01)$. The data demonstrated that transfection of sense hsa-miR-125a-3p effectively inhibited the cell migration and invasion of A549 lung cancer cells.

Transfection of sense miR-125a-3p promotes the expression of tumor suppressor p53 and inhibits the expression of MDM-2.

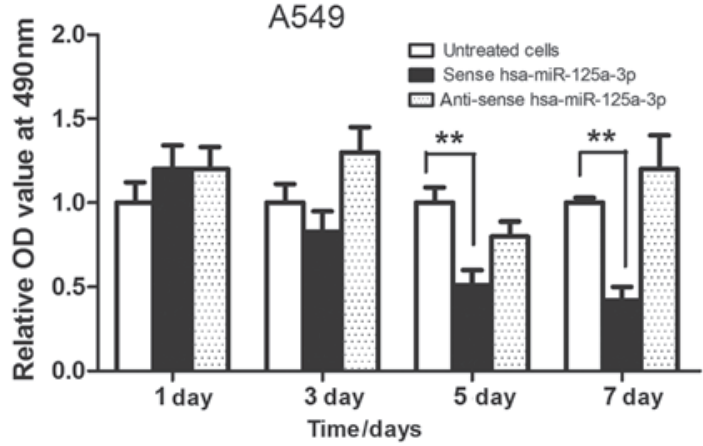

Figure 2. Overexpression of hsa-miR-125a-3p inhibits the proliferation of A549 cells. The lung cancer cells were planted into 48 -well plates. The cells were cultured for $8 \mathrm{~h}$, sense hsa-miR-125a-3p and anti-sense hsa-miR-125a-3p was transfected into A549 lung cancer cells, and cultured for 1, 3, 5 and 7 days, respectively. An MTT assay was used to test the cell proliferation of A549 cells. The untreated cells were used as negative controls. ${ }^{* * *} \mathrm{P}<0.01$.

To further investigate the mechanism of hsa-miR-125a-3p the regulation of apoptosis of lung cancer cells, western blot analysis was used to detect the expression of p53 and MDM-2. As shown in Fig. 5, transfection of sense miR-125a-3p increased the expression of p53 and inhibited the expression of MDM-2 in A549 cells. This is consistent with that observed in the NCI-H460 lung cancer cell line. Untreated cells and the cells transfected with scrambled miRNA were used as negative controls. These results suggested that hsa-miR-125a-3p could promote cell apoptosis by inhibiting the expression of MDM-2 to upregulate the expression of tumor suppressor p53.

\section{Discussion}

miRNA is an endogenous small RNA, which are not encoded into proteins (29). It is well conserved in eukaryotic organisms and is hypothesized to be a vital and evolutionarily component of genetic regulation (30). Studies have demonstrated that miR-125a is important in carcinogenesis (31). Bi et al (32) demonstrated that decreased miR-125a was observed in HCC tissues and cell lines, and it was associated with aggressive pathological features. It was also reported that miR-125a-3p was downregulated in human gastric cancer, and the low expression levels of miR-125a-3p were associated with indicators of enhanced malignant potential, such as tumor size, tumor invasion, lymph node metastasis, liver metastasis, peritoneal dissemination, advanced clinical stage and poor prognosis (33).

In the present study, the role of hsa-miR-125a-3p was observed in the proliferation and metastasis of lung cancer cells. Firstly, the expression of hsa-miR-125a-3p in different lung cancer cell lines was detected. The results demonstrated that relative mRNA levels of hsa-miR-125a-3p are decreased in A549, NCI-H460 and SPCA-1 lung cancer cell lines compared with the HBE cell line, which suggested hsa-miR-125a-3p may act as a tumor suppressor gene to inhibit the tumor progression. Subsequently, the miR-125a-3p was overexpressed in lung cancer cells to detect the role of miR-125a-3p on the proliferation of A549 cells. The results showed that overexpression of sense hsa-miR-125a-3p inhibited the growth of A549 cells; however, transfection of 
A
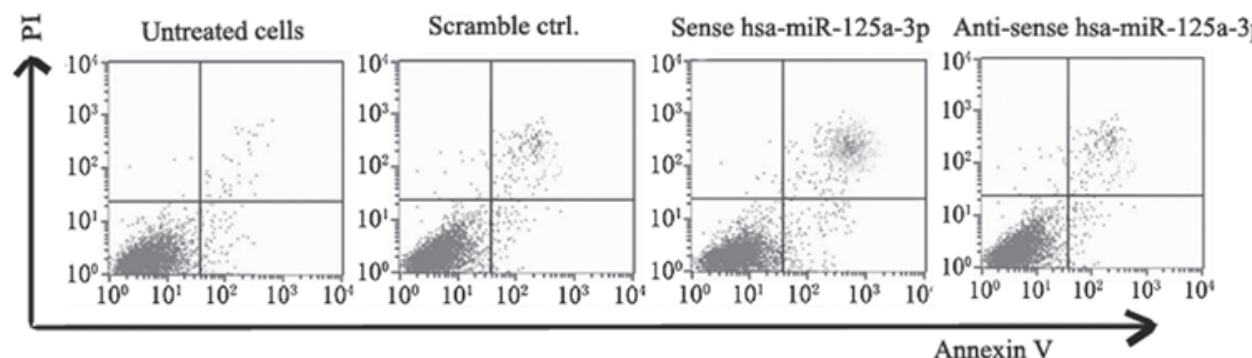

B

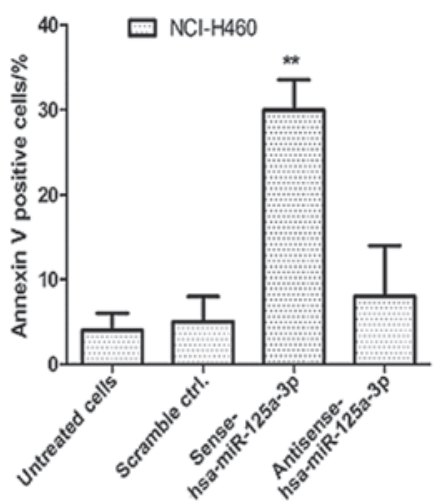

Figure 3. Apoptosis rate increases in NCI-H460 lung cancer cells transfected with sense has-miR-125a-3p. (A) NCI-H460 lung cancer cells were planted onto a 6 -well plate. After $6 \mathrm{~h}$, the cells were transfected with sense hsa-miR-125a-3p, anti-sense miR-125a-3p and scrambled miRNA, respectively. After 5 days, cell apoptosis was detected by annexin V-FITC/PI dual staining analysis. (B) Histogram of the cell apoptosis rate. Untreated cells or the cells transfected with scrambled miRNA were used as negative controls. ${ }^{* *} \mathrm{P}<0.01$ vs. untreated cells and cells transfected with scrambled miRNA. miR, microRNA; FITC, fluorescein isothiocyanate; PI, propidium iodide.

A

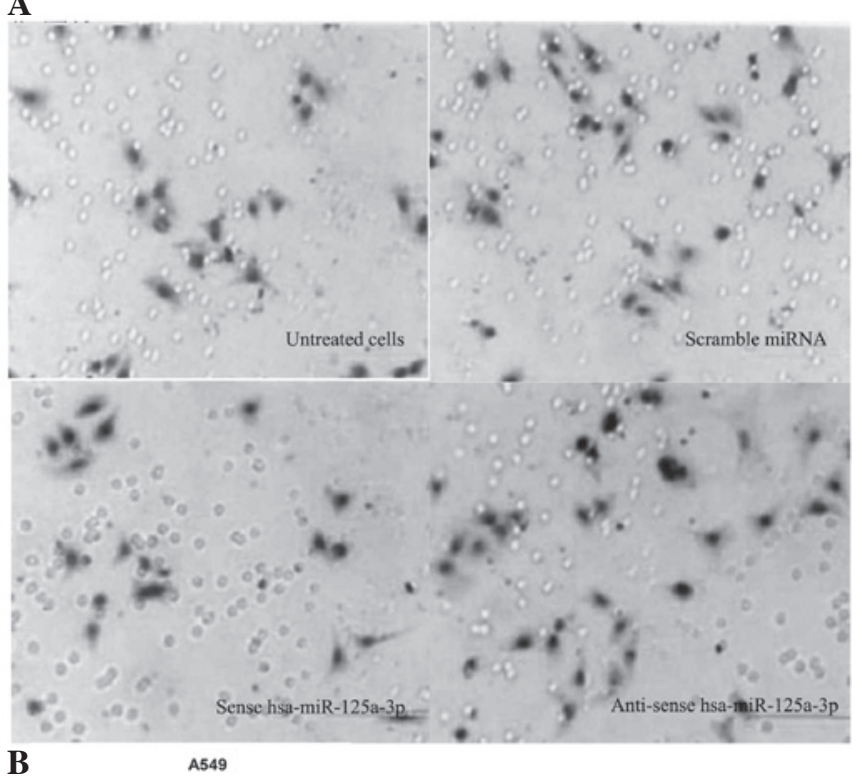

B

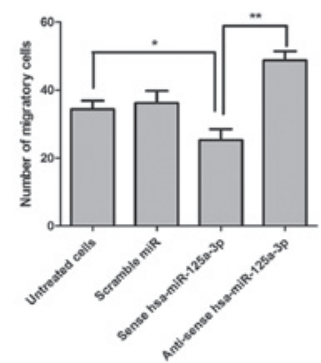

Figure 4. Transfection of sense hsa-miR-125a-3p inhibits the cell migration and invasion of A549 lung cancer cells. (A) Number of migrated A549 cells were stained by hematoxylin in cells transfected with sense hsa-miR-125a-3p, anti-sense hsa-miR-125a-3p and scrambled miRNA. Untreated cells were used as negative controls. Magnification, x200. (B) Histogram showing the number of migrated cells in different groups.** $\mathrm{P}<0.01$ vs. untreated cells.

anti-sense hsa-miR-125a-3p could not suppress the proliferation of lung cancer cells. In addition, transfection of sense miR-125a-3p could enhance the apoptosis rate of NCI-H460 cells, which was detected by annexin V-FITC/PI dual staining analysis.

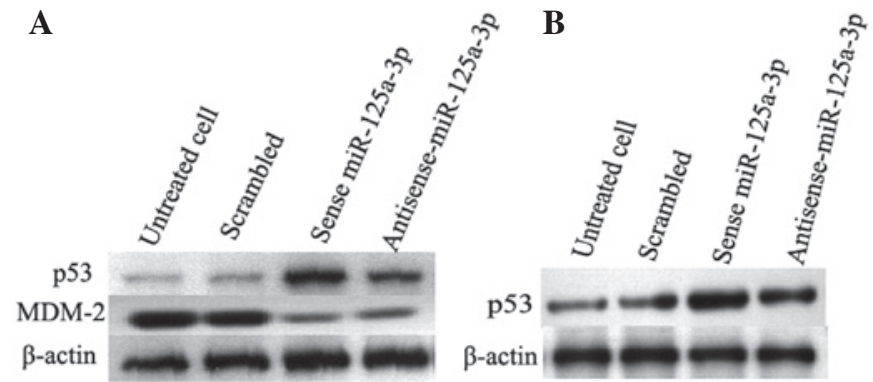

Figure 5. Transfection of sense miR-125a-3p promotes the expression of tumor suppressor p53 and inhibits the expression of MDM-2. Lung cancer (A) A549 cells and (B) NCI-H460 cells were transfected with sense miR-125a-3p, anti-sense miR-125a-3p and scrambled miR for three days. The cell lysates were used to detect the expression of $\mathrm{p} 53$ and MDM-2 by western blot analysis. $\beta$-actin was used as an internal reference and untreated cells were used as negative controls.

Metastasis of tumor cells is one of the predominant reasons for the high mortality rate in lung cancer patients $(13,34)$. Thus, the present study analyzed whether overexpression of sense miR-125a-3p could inhibit the invasion and migration of lung cancer cells. The Transwell migration assay was performed and the data demonstrated that the number of migrated cells in the sense miR-125a-3p transfected group was less than that of the control group, suggesting transfection of sense hsa-miR-125a-3p could inhibit the cell migration and invasion of lung cancer cells. Mdm2 acts as a p53 tumor suppressor and inhibits the transcriptional activity of p53 by regulating its stability. The results showed that transfection of sense miR-125a-3p could inhibit the expression of MDM-2 and promote the expression of tumor suppressor p53. Thus, it may exhibit an antitumor role in lung cancer cells by regulating the MDM-2/p53 signaling pathway. In conclusion, the results demonstrated that upregulation of hsa-miR-125a-3p may be a promising approach for lung cancer therapy.

\section{Acknowledgements}

This study was supported by grants from the Youth Innovation Fund of the Afflilated Hospital of Zhengzhou University. 


\section{References}

1. Xu X, Yang X, Xing C, Zhang S and Cao J: miRNA: The nemesis of gastric cancer (Review). Oncol Lett 6: 631-641, 2013.

2. Cai Y, Yu X, Hu S and Yu J: A brief review on the mechanisms of miRNA regulation. Genomics Proteomics Bioinformatics 7: 147-154, 2009.

3. Lodewijk L, Prins AM, Kist JW, et al: The value of miRNA in diagnosing thyroid cancer: a systematic review. Cancer Biomark 11: 229-238, 2012.

4. Srivastava K and Srivastava A: Comprehensive review of genetic association studies and meta-analyses on miRNA polymorphisms and cancer risk. PLoS ONE 7: e50966, 2012.

5. Scott GK, Goga A, Bhaumik D, Berger CE, Sullivan CS and Benz CC: Coordinate suppression of ERBB2 and ERBB3 by enforced expression of micro-RNA miR-125a or miR-125b. J Biol Chem 282: 1479-1486, 2007.

6. Jiang L, Zhang Q, Chang H, Qiu X and Wang E: hsa-miR-125a-5p enhances invasion in non-small cell lung carcinoma cell lines by upregulating rock-1. Zhongguo Fei Ai Za Zhi 12: 1069-1073, 2009 (In Chinese).

7. Huang B, Luo W, Sun L, et al: MiRNA-125a-3p is a negative regulator of the RhoA-actomyosin pathway in A549 cells. Int J Oncol 42: 1734-1742, 2013.

8. Li X, Sundquist J, Zöller B and Sundquist K: Neighborhood deprivation and lung cancer incidence and mortality: a multilevel analysis from sweden. J Thorac Oncol 10 256-263, 2015.

9. Janković M, Samarzija M, Jakopović M, Kulis T and Znaor A: Trends in lung cancer incidence and mortality in Croatia, 1988-2008. Croat Med J 53: 93-99, 2012.

10. Ondrusova M, Muzik J, Hunakova L, et al: Trends in the lung cancer incidence and mortality in the Slovak and Czech Republics in the contexts of an international comparison. Clin Trans Oncol 659-666, 2012

11. Song Y and Yao Y: Lung cancer screening:review and prospect. Zhonghua Jie He He Hu Xi Za Zhi 37: 164-166, 2014 (In Chinese)

12. Pendharkar D, Ausekar BV and Gupta S: Molecular biology of lung cancer-a review. Indian J Surg Oncol 4: 120-124, 2013.

13. Di JZ, Peng JY and Wang ZG: Prevalence, clinicopathological characteristics, treatment, and prognosis of intestinal metastasis of primary lung cancer: A comprehensive review. Surg Oncol 23: 72-80, 2014

14. Thunnissen E, van der Oord K and den Bakker M: Prognostic and predictive biomarkers in lung cancer. A review. Virchows Arch 464: 347-358, 2014

15. Kumar S, Mohan A and Guleria R: Prognostic implications of circulating anti-p53 antibodies in lung cancer - a review. Eur J Cancer Care (Engl) 18: 248-254, 2009.

16. Bergqvist M: Radiosensitivity in lung cancer with focus on $\mathrm{p} 53$ : a review based on a doctoral thesis. Ups J Med Sci 108: 87-127, 2003.

17. Wang AL, Liu ZX, Li G and Zhang LW: Expression and significance of P53 protein and MDM-2 protein in human gliomas. Chin Med J (Engl) 124: 2530-2533, 2011.
18. Asher G, Lotem J, Sachs L, Kahana C and Shaul Y: Mdm-2 and ubiquitin-independent $\mathrm{p} 53$ proteasomal degradation regulated by NQO1. Proc Natl Acad Sci USA 99: 13125-13130, 2002.

19. Jabbur JR, Tabor AD, Cheng X, et al: Mdm-2 binding and TAF(II)31 recruitment is regulated by hydrogen bond disruption between the p53 residues Thr18 and Asp21. Oncogene 21: 7100-7113, 2002.

20. Almog N, Milyavsky M, Stambolsky P, Falcovitz A, Goldfinger N and Rotter V: The role of the $\mathrm{C}^{\prime}$ terminus of murine p53 in the p53/mdm-2 regulatory loop. Carcinogenesis 22: 779-785, 2001.

21. Kondo I, Iida S, Takagi Y and Sugihara K: MDM2 mRNA expression in the p53 pathway may predict the potential of invasion and liver metastasis in colorectal cancer. Dis Colon Rectum 51: 1395-1402, 2008

22. Nishitani H, Sugimoto N, Roukos V, et al: Two E3 ubiquitin ligases, SCF-Skp2 and DDB1-Cul4, target human Cdt1 for proteolysis. EMBO J 25: 1126-1136, 2006.

23. Peng L, Xu Z, Zhou Y, Yang T, Liang ZQ and Zhang M: Effect of rosiglitazone on cells cycle, apoptosis and expression of Skp2 and p27Kip1 in hepatocellular carcinoma cell line. Zhonghua Gan Zang Bing Za Zhi 18: 148-149, 2010 (In Chinese)

24. Schulman BA, Carrano AC, Jeffrey PD, et al: Insights into SCF ubiquitin ligases from the structure of the Skp1-Skp2 complex. Nature 408: 381-386, 2000

25. Krishan A: Rapid flow cytofluorometric analysis of mammalian cell cycle by propidium iodide staining. J Cell Biol 66: 188-193, 1975.

26. Buchegger F, Dupertuis YM and Perillo-Adamer F: A pitfall of propidium iodide staining in fluorescence-activated cell sorting cell cycle analysis? Cancer Res 67: 5576-5577, 2007.

27. Rüster B, Grace B, Seitz O, Seifried E and Henschler R: Induction and detection of human mesenchymal stem cell migration in the 48-well reusable transwell assay. Stem Cells Dev 14: 231-235, 2005.

28. Primiceri E, Chiriacò MS, Dioguardi F, et al: Automatic transwell assay by an EIS cell chip to monitor cell migration. Lab Chip 11: 4081-4086, 2011.

29. Tilghman SL, Rhodes LV, Bratton MR, et al: Phytoalexins, miRNAs and breast cancer: a review of phytochemical-mediated miRNA regulation in breast cancer. J Health Care Poor Underserved 24 (Suppl): 36-46, 2013.

30. Kim JK, Noh JH, Jung KH, et al: Sirtuin7 oncogenic potential in human hepatocellular carcinoma and its regulation by the tumor suppressors MiR-125a-5p and MiR-125b. Hepatology 57: 1055-1067, 2013.

31. Jiang L, Huang Q, Chang J, Wang E and Qiu X: MicroRNA HSA-miR-125a-5p induces apoptosis by activating p53 in lung cancer cells. Exp Lung Res 37: 387-398, 2011.

32. Bi Q, Tang S, Xia L, et al: Ectopic expression of MiR-125a inhibits the proliferation and metastasis of hepatocellular carcinoma by targeting MMP11 and VEGF. PLoS ONE 7: e40169, 2012.

33. Hashiguchi Y, Nishida N, Mimori K, et al: Down-regulation of miR-125a-3p in human gastric cancer and its clinicopathological significance. Int J Oncol 40: 1477-1482, 2012.

34. Collins J, Noble S, Chester J, Coles B and Byrne A: The assessment and impact of sarcopenia in lung cancer: a systematic literature review. BMJ Open 4: e003697, 2014 